TRANSACTIONS OF THE

AMERICAN MATHEMATICAL SOCIETY

Volume 360, Number 12, December 2008, Pages 6251-6267

S 0002-9947(08)04613-8

Article electronically published on July 21, 2008

\title{
THE ZERO SET OF SEMI-INVARIANTS FOR EXTENDED DYNKIN QUIVERS
}

\author{
CH. RIEDTMANN AND G. ZWARA
}

\begin{abstract}
We show that the set of common zeros $\mathcal{Z}_{Q, \mathbf{d}}$ of all semi-invariants vanishing at 0 on the variety $\operatorname{rep}(Q, \mathbf{d})$ of all representations with dimension vector $\mathbf{d}$ of an extended Dynkin quiver $Q$ under the group $\mathrm{GL}(\mathbf{d})$ is a complete intersection if $\mathbf{d}$ is "big enough". In case $\operatorname{rep}(Q, \mathbf{d})$ does not contain an open $\mathrm{GL}(\mathbf{d})$-orbit, which is the case not considered so far, the number of irreducible components of $\mathcal{Z}_{Q, \mathbf{d}}$ grows with $\mathbf{d}$, except if $Q$ is an oriented cycle.
\end{abstract}

\section{INTRODUCTION AND MAIN RESUlt}

1.1. Let $k$ be an algebraically closed field, and let $Q=\left(Q_{0}, Q_{1}, t, h\right)$ be a quiver with $n$ vertices and a finite set $Q_{1}$ of arrows $\alpha: t \alpha \rightarrow h \alpha$, where $t \alpha$ and $h \alpha$ denote the tail and the head of $\alpha$, respectively.

A representation of $Q$ over $k$ is a collection $\left(X(i) ; i \in Q_{0}\right)$ of finite dimensional $k$-vector spaces together with a collection $\left(X(\alpha): X(t \alpha) \rightarrow X(h \alpha) ; \alpha \in Q_{1}\right)$ of $k$-linear maps. A morphism $f: X \rightarrow Y$ between two representations is a collection $(f(i): X(i) \rightarrow Y(i))$ of $k$-linear maps such that

$$
f(h \alpha) \circ X(\alpha)=Y(\alpha) \circ f(t \alpha) \quad \text { for all } \alpha \in Q_{1} .
$$

By $\sigma(X)$ we denote the number of pairwise non-isomorphic indecomposable direct summands occurring in a decomposition of $X$ into indecomposables. According to the theorem of Krull-Schmidt, $\sigma(X)$ is well-defined. The dimension vector of a representation $X$ of $Q$ is the vector

$$
\operatorname{dim} X=(\operatorname{dim} X(i))_{i \in Q_{0}} \in \mathbb{N}^{Q_{0}} .
$$

We denote the category of representations of $Q$ by $\operatorname{rep}(Q)$, and for any vector $\mathbf{d}=\left(d_{i}\right)_{i \in Q_{0}} \in \mathbb{N}^{Q_{0}}$,

$$
\operatorname{rep}(Q, \mathbf{d})=\prod_{\alpha \in Q_{1}} \operatorname{Mat}\left(d_{h \alpha} \times d_{t \alpha}, k\right)
$$

is the vector space of representations $X$ of $Q$ with $X(i)=k^{d_{i}}, i \in Q_{0}$. The group

$$
\mathrm{GL}(\mathbf{d})=\prod_{i \in Q_{0}} \mathrm{GL}\left(d_{i}, k\right)
$$

Received by the editors October 12, 2006.

2000 Mathematics Subject Classification. Primary 14L24; Secondary 16G20.

Key words and phrases. Semi-invariants, quivers, representations.

The second author gratefully acknowledges support from the Polish Scientific Grant KBN No. 1 P03A 01827 and the Swiss Science Foundation. 
acts on $\operatorname{rep}(Q, \mathbf{d})$ by

$$
\left(\left(g_{i}\right)_{i \in Q_{0}} \star X\right)(\alpha)=g_{h \alpha} \circ X(\alpha) \circ g_{t \alpha}^{-1} .
$$

Note that the $\mathrm{GL}(\mathbf{d})$-orbit of $X$ consists of the representations $Y$ in $\operatorname{rep}(Q, \mathbf{d})$ which are isomorphic to $X$.

A regular function $f \in k[\operatorname{rep}(Q, \mathbf{d})]$ is called a semi-invariant if, for any $g \in$ $\mathrm{GL}(\mathbf{d}), g \star f=\chi(g) f$ for some group homomorphism $\chi: \mathrm{GL}(\mathbf{d}) \rightarrow k^{*}$ which is a regular function on $\operatorname{GL}(\mathbf{d})$, the so-called weight of $f$. Note that the $k$-algebra generated by all semi-invariants is just the algebra $k[\operatorname{rep}(Q, \mathbf{d})]^{\mathrm{SL}(\mathbf{d})}$ of polynomial functions which are invariant under the product

$$
\mathrm{SL}(\mathbf{d})=\prod_{i \in Q_{0}} \mathrm{SL}\left(d_{i}, k\right) .
$$

Indeed, the algebra $k[\operatorname{rep}(Q, \mathbf{d})]^{\mathrm{SL}(\mathbf{d})}$ is the direct sum of the spaces of $\operatorname{GL}(\mathbf{d})$-semiinvariants of weight $\chi$, where $\chi$ ranges over all characters of GL(d).

In case $\mathbf{d}$ is a sincere prehomogeneous dimension vector, i.e. if $d_{i}>0$ for all $i$ and if the orbit $\operatorname{GL}(\mathbf{d}) \star T$ of some representation $T$ is open and dense, the algebra $k[\operatorname{rep}(Q, \mathbf{d})]^{\mathrm{SL}(\mathbf{d})}$ is a polynomial algebra in $n-\sigma(T)$ generators. In fact, the generators correspond bijectively to the simple objects in the perpendicular category $T^{\perp}$, the full subcategory of $\operatorname{rep}(Q)$ whose objects $Y$ satisfy $\operatorname{Hom}_{Q}(T, Y)=$ $\operatorname{Ext}_{Q}^{1}(T, Y)=0$ [12. We showed in 8 that the variety of common zeros $\mathcal{Z}_{Q, \mathbf{d}}$ of all non-constant semi-invariants is a complete intersection if each of the pairwise non-isomorphic indecomposable direct summands $T_{i}$ of

$$
T=\bigoplus_{i=1}^{\sigma(T)} T_{i}^{\lambda_{i}}
$$

arises with a sufficiently large multiplicity $\lambda_{i}$. Choosing $\lambda_{i}$ larger still, we obtain that $\mathcal{Z}_{Q, \mathbf{d}}$ is irreducible. By [9], $\mathcal{Z}_{Q, \mathbf{d}}$ is a complete intersection or irreducible, for $\lambda_{i} \geq 3$ or $\lambda_{i} \geq 4$, respectively, if $Q$ is a tame quiver, i.e. a disjoint union of Dynkin quivers and extended Dynkin quivers. Chang and Weyman, the first to consider this question, showed in 2 that $\mathcal{Z}_{Q, \mathbf{d}}$ is a complete intersection for any $\mathbf{d}$ if $Q$ is a Dynkin quiver of type $\mathbb{A}_{n}$.

The interest in knowing when $k[\operatorname{rep}(Q, \mathbf{d})]^{\mathrm{SL}(\mathbf{d})}$ is non-singular (or equivalently is a polynomial ring), and when $\mathcal{Z}_{Q, \mathbf{d}}$ is a complete intersection comes from the following fact: Assume a reductive algebraic group $G$ acts regularly and linearly on a finite dimensional $\mathbb{C}$-vector space $V$. The action of $G$ on $V$ is called

(i) coregular if the algebraic quotient $5 \mathrm{5} / / G$ has no singularities,

(ii) cofree if $\mathbb{C}[V]$ is a free module over the invariant ring $\mathbb{C}[V]^{G}$,

(iii) equidimensional if the dimension of $V / / G$ equals the codimension in $V$ of the set of common zeros of all $G$-invariants which vanish at 0 .

G. Schwarz proved in [16] that an action is cofree if and only if it is coregular and equidimensional. He classified all coregular and cofree representations of connected simple algebraic groups ([14, [15]). In [7, P. Littelmann classified all coregular and cofree irreducible representations of semisimple groups.

We have recalled above that in case $\mathbf{d}$ is a prehomogeneous dimension vector the action of $\operatorname{SL}(\mathbf{d})$ on $\operatorname{rep}(Q, \mathbf{d})$ is always coregular, and it is equidimensional if $\mathbf{d}$ is "big enough". 
Unfortunately, most dimension vectors fail to be prehomogeneous, except for Dynkin quivers, in which case they all are. The algebra $k[\operatorname{rep}(Q, \mathbf{d})]^{\mathrm{SL}(\mathbf{d})}$ for an arbitrary pair $(Q, \mathbf{d})$ is not known. If $Q$ is an extended Dynkin quiver, however, Skowroński and Weyman have obtained in [17] a complete description of $k[\operatorname{rep}(Q, \mathbf{d})]^{\mathrm{SL}(\mathbf{d})}$ by generators and relations for an arbitrary dimension vector $\mathbf{d}$. It turns out that in most cases the action of $\operatorname{SL}(\mathbf{d})$ on $\operatorname{rep}(Q, \mathbf{d})$ is coregular (compare 1.3).

Our purpose in the present paper is to study $\mathcal{Z}_{Q, \mathbf{d}}$ for an extended Dynkin quiver and an arbitrary dimension vector. We find that, as in the prehomogeneous case, the action of $\operatorname{SL}(\mathbf{d})$ on $\operatorname{rep}(Q, \mathbf{d})$ is equidimensional if $\mathbf{d}$ is "big enough". But $\mathcal{Z}_{Q, \mathbf{d}}$ does not become irreducible with growing $\mathbf{d}$. In fact, except for the oriented cycle, the number of its irreducible components increases with $\mathbf{d}$.

1.2. From now on until the end of Section 4, we assume that $Q$ does not contain oriented cycles. We will usually not repeat this assumption. We will treat the oriented cycle separately in Section 5 .

We need to recall a few facts and definitions, mostly from [11, before we can state our results. For a quiver $Q$, the Euler bilinear form $\langle-,-\rangle: \mathbb{Z}^{Q_{0}} \times \mathbb{Z}^{Q_{0}} \rightarrow \mathbb{Z}$ is defined by $\langle\mathbf{x}, \mathbf{y}\rangle=\sum_{i \in Q_{0}} x_{i} y_{i}-\sum_{\alpha \in Q_{1}} x_{t \alpha} y_{h \alpha}$. The associated quadratic form $q_{Q}: \mathbb{Z}^{Q_{0}} \rightarrow \mathbb{Z}$, given by $q_{Q}(\mathbf{x})=\langle\mathbf{x}, \mathbf{x}\rangle$, is the Euler quadratic form. If $X$ and $Y$ are representations of $Q$, we have

$$
\langle\operatorname{dim} X, \operatorname{dim} Y\rangle=[X, Y]-{ }^{1}[X, Y]
$$

where we set $[X, Y]=\operatorname{dim}_{k} \operatorname{Hom}_{Q}(X, Y)$ and ${ }^{1}[X, Y]=\operatorname{dim}_{k} \operatorname{Ext}_{Q}^{1}(X, Y)$.

Assume that $Q$ is an extended Dynkin quiver. Then the Euler quadratic form is positive semi-definite, and its radical is $\mathbb{Z} \mathbf{h}$ for a unique vector $\mathbf{h} \in \mathbb{N}^{Q_{0}}$. The defect is the linear form $\partial: \mathbb{Z}^{Q_{0}} \rightarrow \mathbb{Z}$ given by $\partial(\mathbf{x})=\langle\mathbf{h}, \mathbf{x}\rangle=-\langle\mathbf{x}, \mathbf{h}\rangle$. A representation $X$ is called preprojective, regular, or preinjective, if $\partial(\operatorname{dim} U)<0$, $=0$, or $>0$, respectively, for every indecomposable direct summand $U$ of $X$. Any representation $X$ has a unique decomposition $X=X_{\mathcal{P}} \oplus X_{\mathcal{R}} \oplus X_{\mathcal{I}}$, where $X_{\mathcal{P}}, X_{\mathcal{R}}$, $X_{\mathcal{I}}$ are preprojective, regular, and preinjective, respectively.

The regular representations form an exact abelian subcategory $\mathcal{R}$ of $\operatorname{rep}(Q)$. The category $\mathcal{R}$ decomposes into a $\mathbb{P}^{1}(k)$-family $\coprod_{\mu \in \mathbb{P}^{1}(k)} \mathcal{R}_{\mu}$ of uniserial categories. For each $\mu$, the category $\mathcal{R}_{\mu}$ contains a finite number $r_{\mu}$ of simple objects; their dimension vectors add up to $\mathbf{h}$. The set $\mathcal{E}=\left\{\mu \in \mathbb{P}^{1}(k) ; r_{\mu}>1\right\}$ has at most three elements. For $\mu \notin \mathcal{E}$, we denote the unique simple representations of $\mathcal{R}_{\mu}$ by $H_{\mu}$. A simple regular representation with dimension vector $\mathbf{h}$ is called homogeneous. We have $\sum\left(r_{\mu}-1\right)=\# Q_{0}-2=n-2$.

If $\mathbf{d}$ is not a prehomogeneous dimension vector, then representations in $\operatorname{rep}(Q, \mathbf{d})$ are necessarily generically regular. In fact, $\operatorname{rep}(Q, \mathbf{d})$ contains an open subset consisting of representations $H_{\mu_{1}} \oplus \cdots \oplus H_{\mu_{p}} \oplus V$, where $p \geq 1$ and $\mu_{1}, \ldots, \mu_{p} \notin \mathcal{E}$ are pairwise different, any indecomposable direct summand of $V$ belongs to $\mathcal{R}_{\mu}$ for some $\mu \in \mathcal{E}$, and $\operatorname{dim} V=\mathbf{e}$ is prehomogeneous. We call the decomposition $\mathbf{d}=p \cdot \mathbf{h}+\mathbf{e}$ the canonical decomposition of $\mathbf{d}$.

1.3. We are now ready to state our results.

As a consequence of the theorem of Skowroński and Weyman [17, Thm.21], we know that the algebra $k[\operatorname{rep}(Q, \mathbf{d})]^{\mathrm{SL}(\mathbf{d})}$ is a polynomial ring if $Q$ is an extended Dynkin quiver, $\mathbf{d}=p \cdot \mathbf{h}+\mathbf{e}$ is not prehomogeneous and $p \geq 2$. If $Q$ is of type 
$\widetilde{\mathbb{A}}_{n-1}$, this is true even if $p=1$. Moreover, if the algebra $k[\operatorname{rep}(Q, \mathbf{d})]^{\operatorname{SL}(\mathbf{d})}$ is not a polynomial algebra, the categorical quotient $\operatorname{rep}(Q, \mathbf{d}) / / \mathrm{SL}(\mathbf{d})$ is singular. Indeed, its coordinate ring is of the form

$$
k\left[X_{1}, \ldots, X_{a}, Y_{1}, \ldots, Y_{b}, Z_{1}, \ldots, Z_{c}\right] /(f),
$$

for some polynomial $f=X_{1} \cdots X_{a^{\prime}}+Y_{1} \cdots Y_{b^{\prime}}+Z_{1} \cdots Z_{c^{\prime}}$ with $a^{\prime} \leq a, b^{\prime} \leq b$, $c^{\prime} \leq c$, and $a^{\prime}, b^{\prime}, c^{\prime} \geq 2$, and $f$ has a singularity at 0 .

Theorem 1. Let $Q$ be an extended Dynkin quiver different from the oriented cycle, $\mathbf{d} \in \mathbb{N}^{Q_{0}}$ a non-prehomogeneous dimension vector with canonical decomposition $\mathbf{d}=p \cdot \mathbf{h}+\mathbf{e}$, and $V=\bigoplus_{i=1}^{\sigma(V)} V_{i}^{\lambda_{i}}$ a representation in the open orbit of $\operatorname{rep}(Q, \mathbf{e})$ with $V_{i}$ indecomposable and pairwise non-isomorphic. Assume that either $Q$ is of type $\widetilde{\mathbb{A}}_{n-1}$ or else $p \geq 3$ and $\lambda_{i} \geq 3$ for all $i$. Then we have:

(i) The action of $\mathrm{SL}(\mathbf{d})$ on $\operatorname{rep}(Q, \mathbf{d})$ is equidimensional.

(ii) Each irreducible component of $\mathcal{Z}_{Q, \mathbf{d}}$ is the closure of a $\mathrm{GL}(\mathbf{d})$-orbit.

(iii) The number of irreducible components of $\mathcal{Z}_{Q, \mathbf{d}}$ is at least $p-2$.

Remark 1.1. Note that the behavior of the number of irreducible components of $\mathcal{Z}_{Q, \mathbf{d}}$ is quite different from what happens for $\mathbf{d}$ prehomogeneous, since in that case $\mathcal{Z}_{Q, N \cdot \mathbf{d}}$ is irreducible for $N$ large.

Remark 1.2. If $Q$ is of type $\widetilde{\mathbb{D}}_{n-1}$, it can be shown that the assumption on $\lambda_{i}$ may be dropped if $p \geq 4$. We do not know if such a tradeoff is possible if $Q$ is of type $\widetilde{\mathbb{E}}_{6}, \widetilde{\mathbb{E}}_{7}$, or $\widetilde{\mathbb{E}}_{8}$.

Remark 1.3. Our arguments do not extend to the case $\mathbf{d}=2 \cdot \mathbf{h}$. Indeed, for

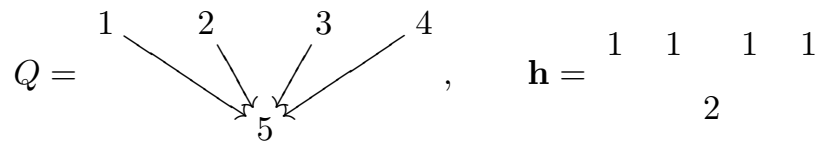

the algebra $k[\operatorname{rep}(Q, \mathbf{d})]^{\mathrm{SL}(2 \cdot \mathbf{h})}$ is a polynomial ring in 6 variables by [17, Thm. 17], but $\mathcal{Z}_{Q, \mathbf{d}}$ contains the representation $X=X_{1} \oplus X_{2}$, where $X_{1}$ is the one-dimensional representation supported at the vertex 5 and the orbit of the representation $X_{2}$ is open in $\operatorname{rep}\left(Q, 2 \cdot \mathbf{h}-\operatorname{dim} X_{1}\right)$, and

$$
\operatorname{codim} \overline{\mathrm{GL}(2 \cdot \mathbf{h}) \star X}={ }^{1}[X, X]={ }^{1}\left[X_{2}, X_{1}\right]=5 .
$$

The paper is organized as follows: In Section 2 we generalize Schofield's result relating semi-invariants to objects in some perpendicular category [12] to nonprehomogeneous dimension vectors. In Section 3 we describe these generalized perpendicular categories, and we prove our main result in Section 4 . The last section is devoted to the oriented cycle. As in that case there exist non-constant GL(d)invariants on $\operatorname{rep}(Q, \mathbf{d})$, and we need to modify the description of $\mathcal{Z}_{Q, \mathbf{d}}$ slightly. We obtain the following corollary.

Corollary 1.4. If $Q$ is an $\widetilde{\mathbb{A}}_{n-1}$-quiver, then $\mathcal{Z}_{Q, \mathbf{d}}$ is a complete intersection for any dimension vector $\mathbf{d}$. 


\section{SEMI-INVARIANTS AND PERPENDICULAR CATEGORIES}

2.1. Let $Q$ be a quiver, $\mathbf{d}, \mathbf{e} \in \mathbb{N}^{Q_{0}}$, and let $X, Y$ be representations of $Q$ with $\operatorname{dim} X=\mathbf{d}$ and $\operatorname{dim} Y=\mathbf{e}$. Consider the linear map

$$
\mathcal{F}_{X, Y}: \bigoplus_{i \in Q_{0}} \operatorname{Hom}_{k}\left(k^{d_{i}}, k^{e_{i}}\right) \rightarrow \bigoplus_{\alpha \in Q_{1}} \operatorname{Hom}_{k}\left(k^{d_{t \alpha}}, k^{e_{h \alpha}}\right)
$$

which sends $\left(g_{i} ; i \in Q_{0}\right)$ to $\left(h_{\alpha} ; \alpha \in Q_{1}\right)$ with $h_{\alpha}=g_{h \alpha} \circ X(\alpha)-Y(\alpha) \circ g_{t \alpha}$. Note that $\operatorname{Ker} \mathcal{F}_{X, Y}=\operatorname{Hom}_{Q}(X, Y)$ and Coker $\mathcal{F}_{X, Y}=\operatorname{Ext}_{Q}^{1}(X, Y)$, which implies that

$$
\langle\mathbf{d}, \mathbf{e}\rangle=[X, Y]-{ }^{1}[X, Y] .
$$

If we assume that $\langle\mathbf{d}, \mathbf{e}\rangle=0$, the linear map $\mathcal{F}_{X, Y}$ will be represented by a square matrix $H_{X, Y}$ (with respect to some bases), and the determinant $\operatorname{det} H_{X, Y}$ is a $\mathrm{GL}(\mathbf{d}) \times \mathrm{GL}(\mathbf{e})$ semi-invariant on $\operatorname{rep}(Q, \mathbf{d}) \times \operatorname{rep}(Q, \mathbf{e})$ by 12 . We denote by $f_{Y} \in k[\operatorname{rep}(Q, \mathbf{d})]$ the semi-invariant associated to a representation $Y$. Note that, for a short exact sequence

$$
0 \rightarrow Y^{\prime} \rightarrow Y \rightarrow Y^{\prime \prime} \rightarrow 0
$$

with $\left\langle\mathbf{d}, \operatorname{dim} Y^{\prime}\right\rangle=\langle\mathbf{d}, \operatorname{dim} Y\rangle=\left\langle\mathbf{d}, \operatorname{dim} Y^{\prime \prime}\right\rangle=0$, we have that $f_{Y}$ is a non-zero multiple of $f_{Y^{\prime}} \cdot f_{Y^{\prime \prime}}$ [3].

The semi-invariant $f_{Y}$ does not vanish identically on $\operatorname{rep}(Q, \mathbf{d})$ if and only if there exists some $T \in \operatorname{rep}(Q, \mathbf{d})$ with $[T, Y]={ }^{1}[T, Y]=0$. We define the perpendicular category $\mathbf{d}^{\perp}$ to be the full subcategory of $\operatorname{rep}(Q)$ whose objects are the representations $Y$ of $Q$ for which there is a $T \in \operatorname{rep}(Q, \mathbf{d})$ with $[T, Y]={ }^{1}[T, Y]=0$. Note that in this case $[X, Y]={ }^{1}[X, Y]=0$ for $X$ in some dense open subset of $\operatorname{rep}(Q, \mathbf{d})$, as $[-, Y]=0$ and ${ }^{1}[-, Y]=0$ are open conditions. In case $\mathbf{d}$ is prehomogeneous, $\mathbf{d}^{\perp}$ is just the category $T^{\perp}$ introduced by Schofield in [12, where $T \in \operatorname{rep}(Q, \mathbf{d})$ lies in the open orbit.

The following result from [3], which in characteristic zero also follows from [13], relates $\mathbf{d}^{\perp}$ to semi-invariants.

Proposition 2.1. If $Q$ does not contain oriented cycles and $\mathbf{d} \in \mathbb{N}^{Q_{0}}$, the functions $f_{Y}, Y \in \mathbf{d}^{\perp}$, span the space $k[\operatorname{rep}(Q, \mathbf{d})]^{\mathrm{SL}(\mathbf{d})}$.

2.2. We start with the following lemma.

Lemma 2.2. $\mathbf{d}^{\perp}$ is an exact abelian subcategory of $\operatorname{rep}(Q)$.

Proof. Obviously $\mathbf{d}^{\perp}$ is closed under taking direct summands. Let $Y^{\prime}$ and $Y^{\prime \prime}$ belong to $\mathbf{d}^{\perp}$. Then there is a $T \in \operatorname{rep}(Q, \mathbf{d})$ for which

$$
\left[T, Y^{\prime}\right]={ }^{1}\left[T, Y^{\prime}\right]=\left[T, Y^{\prime \prime}\right]={ }^{1}\left[T, Y^{\prime \prime}\right]=0 .
$$

Let $f: Y^{\prime} \rightarrow Y^{\prime \prime}$ be a homomorphism. Then we get two induced long exact sequences

$$
\begin{aligned}
0 & \rightarrow \operatorname{Hom}_{Q}(T, \operatorname{Ker}(f)) \rightarrow \operatorname{Hom}_{Q}\left(T, Y^{\prime}\right) \rightarrow \operatorname{Hom}_{Q}(T, \operatorname{Im}(f)) \rightarrow \\
& \rightarrow \operatorname{Ext}_{Q}^{1}(T, \operatorname{Ker}(f)) \rightarrow \operatorname{Ext}_{Q}^{1}\left(T, Y^{\prime}\right) \rightarrow \operatorname{Ext}_{Q}^{1}(T, \operatorname{Im}(f)) \rightarrow 0, \\
0 & \rightarrow \operatorname{Hom}_{Q}(T, \operatorname{Im}(f)) \rightarrow \operatorname{Hom}_{Q}\left(T, Y^{\prime \prime}\right) \rightarrow \operatorname{Hom}_{Q}(T, \operatorname{Coker}(f)) \rightarrow \\
& \rightarrow \operatorname{Ext}_{Q}^{1}(T, \operatorname{Im}(f)) \rightarrow \operatorname{Ext}_{Q}^{1}\left(T, Y^{\prime \prime}\right) \rightarrow \operatorname{Ext}_{Q}^{1}(T, \operatorname{Coker}(f)) \rightarrow 0 .
\end{aligned}
$$


This implies that

$$
\begin{aligned}
& {[T, \operatorname{Ker}(f)]=[T, \operatorname{Im}(f)]={ }^{1}[T, \operatorname{Ker}(f)]={ }^{1}[T, \operatorname{Im}(f)]} \\
& =[T, \operatorname{Coker}(f)]={ }^{1}[T, \operatorname{Coker}(f)]=0 .
\end{aligned}
$$

Hence the subcategory $\mathbf{d}^{\perp}$ is closed under kernels, images and cokernels. If $Y$ is an extension

$$
0 \rightarrow Y^{\prime} \rightarrow Y \rightarrow Y^{\prime \prime} \rightarrow 0
$$

of $Y^{\prime \prime}$ by $Y^{\prime}$, considering the long exact sequence obtained from mapping $T$ to this short exact sequence yields $Y \in \mathbf{d}^{\perp}$.

If $\mathbf{d}$ is prehomogeneous, $\mathbf{d}^{\perp}$ is equivalent to the category of representations of some quiver by [12]. For arbitrary $\mathbf{d}, \mathbf{d}^{\perp}$ may have infinitely many simple objects, however. We will compute $\mathbf{d}^{\perp}$ in case $Q$ is an extended Dynkin quiver and $\mathbf{d}$ is not prehomogeneous in Section 3 ,

2.3. For further reference, we collect a few properties of $\mathbf{d}^{\perp}$.

Proposition 2.3. Let $\mathbf{d}=\mathbf{d}^{\prime}+\mathbf{d}^{\prime \prime}$ with $\mathbf{d}, \mathbf{d}^{\prime}, \mathbf{d}^{\prime \prime} \in \mathbb{N}^{Q_{0}}$, and suppose that generically a representation $T \in \operatorname{rep}(Q, \mathbf{d})$ has a subrepresentation $T^{\prime} \in \operatorname{rep}\left(Q, \mathbf{d}^{\prime}\right)$. Then

$$
\mathbf{d}^{\perp} \cap\left(\mathbf{d}^{\prime}\right)^{\perp}=\left(\mathbf{d}^{\prime \prime}\right)^{\perp} \cap\left(\mathbf{d}^{\prime}\right)^{\perp}=\left(\mathbf{d}^{\prime \prime}\right)^{\perp} \cap \mathbf{d}^{\perp} .
$$

Proof. We only prove the first equality. Suppose $Y \in\left(\mathbf{d}^{\prime \prime}\right)^{\perp} \cap\left(\mathbf{d}^{\prime}\right)^{\perp}$, and choose $T^{\prime} \in \operatorname{rep}\left(Q, \mathbf{d}^{\prime}\right)$ with $\left[T^{\prime}, Y\right]={ }^{1}\left[T^{\prime}, Y\right]=0$ and $T^{\prime \prime} \in \operatorname{rep}\left(Q, \mathbf{d}^{\prime \prime}\right)$ with $\left[T^{\prime \prime}, Y\right]=$ ${ }^{1}\left[T^{\prime \prime}, Y\right]=0$. Then obviously $\left[T^{\prime} \oplus T^{\prime \prime}, Y\right]={ }^{1}\left[T^{\prime} \oplus T^{\prime \prime}, Y\right]=0$, which implies $Y \in$ $\mathbf{d}^{\perp}$. Conversely, if $Y \in \mathbf{d}^{\perp} \cap\left(\mathbf{d}^{\prime}\right)^{\perp}$, choose $T \in \operatorname{rep}(Q, \mathbf{d})$ with $[T, Y]={ }^{1}[T, Y]=0$ and having a subrepresentation $T^{\prime} \subseteq T$ with $\operatorname{dim} T^{\prime}=\mathbf{d}^{\prime}$. Note that ${ }^{1}[T, Y]=0$ implies ${ }^{1}\left[T^{\prime}, Y\right]=0$ as the map $\operatorname{Ext}_{Q}^{1}(T, Y) \rightarrow \operatorname{Ext}_{Q}^{1}\left(T^{\prime}, Y\right)$ is surjective. But as $Y \in\left(\mathbf{d}^{\prime}\right)^{\perp}$ we have

$$
\left\langle\mathbf{d}^{\prime}, \operatorname{dim} Y\right\rangle=\left[T^{\prime}, Y\right]-{ }^{1}\left[T^{\prime}, Y\right]=0
$$

and thus $\left[T^{\prime}, Y\right]=0$. Applying the functor $\operatorname{Hom}_{Q}(-, Y)$ we find $\left[T / T^{\prime}, Y\right]=$ ${ }^{1}\left[T / T^{\prime}, Y\right]=0$ and thus $Y \in\left(\mathbf{d}^{\prime}\right)^{\perp} \cap\left(\mathbf{d}^{\prime \prime}\right)^{\perp}$.

Corollary 2.4. Let $z$ be a sink of $Q$, and denote by $\varepsilon_{z} \in \mathbb{N}^{Q_{0}}$ the vector given by $\varepsilon_{z}(y)=\delta_{z, y}$. For $\mathbf{d} \in \mathbb{N}^{Q_{0}}$, set $\overline{\mathbf{d}}=\mathbf{d}-d_{z} \cdot \varepsilon_{z}$. Then

$$
\left\{Y \in \mathbf{d}^{\perp} ; Y(z)=0\right\}=\left\{Y \in(\overline{\mathbf{d}})^{\perp} ; Y(z)=0\right\} .
$$

Proof. We may assume that $d_{z}>0$. Apply Proposition 2.3 for $\mathbf{d}^{\prime}=d_{z} \cdot \varepsilon_{z}$, and note that

$$
\left(\mathbf{d}^{\prime}\right)^{\perp}=\left(\varepsilon_{z}\right)^{\perp}=\{Y \in \operatorname{rep}(Q) ; Y(z)=0\},
$$

as the one dimensional representation $E_{z}$ supported at $z$ is simple projective, and $\operatorname{Hom}_{Q}\left(E_{z}, Y\right)$ is isomorphic to $Y(z)$.

Proposition 2.5. Let $\mathbf{d}=\mathbf{d}^{\prime}+\mathbf{d}^{\prime \prime}$, and suppose that any $T$ in a dense open set $\mathcal{U} \subseteq \operatorname{rep}(Q, \mathbf{d})$ decomposes as $T=T^{\prime} \oplus T^{\prime \prime}$ for some $T^{\prime} \in \operatorname{rep}\left(Q, \mathbf{d}^{\prime}\right)$ and some $T^{\prime \prime} \in \operatorname{rep}\left(Q, \mathbf{d}^{\prime \prime}\right)$. Then $\mathbf{d}^{\perp}=\left(\mathbf{d}^{\prime}\right)^{\perp} \cap\left(\mathbf{d}^{\prime \prime}\right)^{\perp}$.

Proof. The inclusion $\left(\mathbf{d}^{\prime}\right)^{\perp} \cap\left(\mathbf{d}^{\prime \prime}\right)^{\perp} \subseteq \mathbf{d}^{\perp}$ follows from Proposition 2.3 Conversely, let $Y \in \mathbf{d}^{\perp}$, and choose $T \in \mathcal{U}$ with $[T, Y]={ }^{1}[T, Y]=0$. Then clearly

$$
\left[T^{\prime}, Y\right]={ }^{1}\left[T^{\prime}, Y\right]=\left[T^{\prime \prime}, Y\right]={ }^{1}\left[T^{\prime \prime}, Y\right]=0
$$

if $T=T^{\prime} \oplus T^{\prime \prime}$ is a decomposition with $\operatorname{dim} T^{\prime}=\mathbf{d}^{\prime}$ and $\operatorname{dim} T^{\prime \prime}=\mathbf{d}^{\prime \prime}$. 
2.4. Finally, we wish to study the behavior of $\mathbf{d}^{\perp}$ under reflection functors. Let $z$ be a sink of $Q$, and let $\alpha_{j}: y_{j} \rightarrow z, j=1, \ldots, s$ be the arrows of $Q$ with head $z$. Define a new quiver $Q^{\prime}$, obtained from $Q$ by deleting $z$ and $\alpha_{1}, \ldots, \alpha_{s}$ and by adding a new vertex $z^{\prime}$ as well as arrows $\beta_{j}: z^{\prime} \rightarrow y_{j}, j=1, \ldots, s$. Let $E_{z}$ and $E_{z^{\prime}}^{\prime}$ be the one-dimensional representations of $Q$ and $Q^{\prime}$, supported at $z$ and $z^{\prime}$, respectively. Note that $E_{z}$ is simple projective in $\operatorname{rep}(Q)$ and $E_{z^{\prime}}^{\prime}$ is simple injective in $\operatorname{rep}\left(Q^{\prime}\right)$.

We consider the reflection functor

$$
\mathcal{F}: \operatorname{rep}(Q) \rightarrow \operatorname{rep}\left(Q^{\prime}\right)
$$

associated with $z$. Recall that

$$
(\mathcal{F} X)(i)= \begin{cases}X(i) & i \neq z^{\prime} \\ \operatorname{Ker}\left(\bigoplus X\left(y_{j}\right) \stackrel{\left[X\left(\alpha_{1}\right), \ldots, X\left(\alpha_{s}\right)\right]}{\longrightarrow} X(z)\right) & i=z^{\prime}\end{cases}
$$

and that

$$
(\mathcal{F} X)\left(\beta_{l}\right):(\mathcal{F} X)\left(z^{\prime}\right) \rightarrow(\mathcal{F} X)\left(y_{l}\right)=X\left(y_{l}\right)
$$

is the inclusion of $(\mathcal{F} X)\left(z^{\prime}\right)$ into $\bigoplus_{j=1}^{s} X\left(y_{j}\right)$ followed by the projection to $X\left(y_{l}\right)$ (see [1, 4]). The functor $\mathcal{F}$ restricts to an equivalence

$$
\mathcal{F}:(\operatorname{rep}(Q))^{\prime} \rightarrow\left(\operatorname{rep}\left(Q^{\prime}\right)\right)^{\prime}
$$

from the full subcategory $(\operatorname{rep}(Q))^{\prime}$ of $\operatorname{rep}(Q)$ whose objects do not contain $E_{z}$ as a direct summand, or equivalently have no non-trivial morphisms to $E_{z}$, to the full subcategory $\left(\operatorname{rep}\left(Q^{\prime}\right)\right)^{\prime}$ of $\operatorname{rep}\left(Q^{\prime}\right)$ whose objects do not contain $E_{z^{\prime}}^{\prime}$ as a direct summand.

Suppose $\mathbf{d} \in \mathbb{N}^{Q_{0}}$ satisfies $d_{z}<\sum_{h \alpha=z} d_{t \alpha}$, and define $\mathbf{d}^{\prime} \in \mathbb{Z}^{Q_{0}^{\prime}}$ by

$$
d_{x}^{\prime}= \begin{cases}d_{x}, & x \neq z^{\prime} \\ \sum_{h \alpha=z} d_{t \alpha}-d_{z}, & x=z^{\prime}\end{cases}
$$

Note that $d_{z^{\prime}}^{\prime}>0$. For $T \in(\operatorname{rep}(Q))^{\prime}$ with $\operatorname{dim} T=\mathbf{d}$, we have $\mathcal{F} T \in\left(\operatorname{rep}\left(Q^{\prime}\right)\right)^{\prime}$ with $\operatorname{dim} \mathcal{F} T=\mathbf{d}^{\prime}$.

Proposition 2.6. Let $Q$ be a quiver with a sink $z, \mathbf{d} \in \mathbb{N}^{Q_{0}}$ with $d_{z}<\sum_{h \alpha=z} d_{t \alpha}$, and let $Q^{\prime}, \mathbf{d}^{\prime}$ be defined as above. Then $\mathcal{F}\left(\mathbf{d}^{\perp_{Q}}\right)=\left(\mathbf{d}^{\prime}\right)^{\perp_{Q^{\prime}}}$.

Proof. We will prove that $\mathcal{F} Y \in\left(\mathbf{d}^{\prime}\right)^{\perp_{Q^{\prime}}}$ for $Y \in \mathbf{d}^{\perp_{Q}}$; the other inclusion is obtained from using the reflection functor $\mathcal{F}^{-1}:\left(\operatorname{rep}\left(Q^{\prime}\right)\right)^{\prime} \rightarrow(\operatorname{rep}(Q))^{\prime}$. Choose $T \in \operatorname{rep}(Q, \mathbf{d})$ such that $[T, Y]={ }^{1}[T, Y]=0$ and such that $E_{z}$ is not a direct summand of $T$. This is possible as generically $\left[T, E_{z}\right]=0$ on $\operatorname{rep}(Q, \mathbf{d})$. Note that $E_{z}$ is not a direct summand of $Y$ either as

$$
\left\langle\mathbf{d}, \boldsymbol{\varepsilon}_{z}\right\rangle=-d_{z^{\prime}}^{\prime}=-{ }^{1}\left[T, E_{z}\right]<0 .
$$

But then we have $T, Y \in(\operatorname{rep}(Q))^{\prime}$, and we know

$$
[\mathcal{F} T, \mathcal{F} Y]=[T, Y]=0 \quad \text { and } \quad{ }^{1}[\mathcal{F} T, \mathcal{F} Y]={ }^{1}[T, Y]=0 .
$$

So $\mathcal{F} Y$ belongs to $\left(\mathbf{d}^{\prime}\right)^{\perp_{Q^{\prime}}}$. 


\section{Extended Dynkin QUivers}

3.1. Throughout this section $Q$ is an extended Dynkin quiver. Remember that by $\mathcal{E}$ we denote the set $\mathcal{E}=\left\{\mu \in \mathbb{P}^{1}(k) ; r_{\mu}>1\right\}$, where $r_{\mu}$ is the number of simple objects in the category $R_{\mu}$. We need to recall two more results from [1].

Lemma 3.1. Let $X_{\mathcal{P}}, X_{\mathcal{I}}, X_{\mu}$ be a non-zero preprojective, preinjective and regular representation in $\mathcal{R}_{\mu}$, respectively, $\mu \in \mathbb{P}^{1}(k)$. Then we have

(i) $\left[X_{\mu}, X_{\mathcal{P}}\right]={ }^{1}\left[X_{\mathcal{P}}, X_{\mu}\right]=0$ for all $\mu$,

(ii) $\left[X_{\mathcal{I}}, X_{\mu}\right]={ }^{1}\left[X_{\mu}, X_{\mathcal{I}}\right]=0$ for all $\mu$,

(iii) $\left[X_{\mathcal{I}}, X_{\mathcal{P}}\right]={ }^{1}\left[X_{\mathcal{P}}, X_{\mathcal{I}}\right]=0$,

(iv) $\left[X_{\mu}, X_{\nu}\right]={ }^{1}\left[X_{\mu}, X_{\nu}\right]=0$ for $\mu \neq \nu$,

(v) $\left[X_{\mathcal{P}}, X_{\mu}\right]>0$ and $\left[X_{\mu}, X_{\mathcal{I}}\right]>0$ if $\mu \notin \mathcal{E}$.

3.2. For $r \geq 1$ we denote by $C_{r}$ the oriented cycle with $r$ vertices:

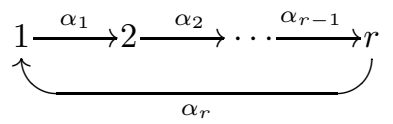

We call a representation $X$ of $C_{r}$ nilpotent if there is a positive integer $N_{X}$ such that $X(\pi)=0$ for any path $\pi$ of length greater than or equal to $N_{X}$.

Lemma 3.2. For $\mu \in \mathbb{P}^{1}(k)$, the category $\mathcal{R}_{\mu}$ is equivalent to the category of nilpotent representations of the oriented cycle $C_{r_{\mu}}$.

Fix a non-prehomogeneous dimension vector $\mathbf{d}$ with canonical decomposition $\mathbf{d}=p \cdot \mathbf{h}+\mathbf{e}, p \geq 1$. We choose $V \in \operatorname{rep}(Q, \mathbf{e})$ such that the $\mathrm{GL}(\mathbf{e})$-orbit of $V$ is open, and we decompose $V=\bigoplus_{\mu \in \mathcal{E}} V_{\mu}, V_{\mu} \in \mathcal{R}_{\mu}$. With this notation we have the following results.

Proposition 3.3. Let $\mathbf{e}_{\mu}=\operatorname{dim} V_{\mu}$ for $\mu \in \mathcal{E}$. Then:

(i) $\mathbf{h}^{\perp}=\coprod_{\mu \in \mathbb{P}^{1}(k)} \mathcal{R}_{\mu}$.

(ii) $\mathbf{d}^{\perp}=\mathbf{h}^{\perp} \cap \mathbf{e}^{\perp}$.

(iii) An indecomposable representation $Y \in \mathcal{R}_{\mu}$ belongs to $\mathbf{e}^{\perp}$ if and only if either $\mu \notin \mathcal{E}$ or, for $\mu \in \mathcal{E}, Y \in\left(\mathbf{e}_{\mu}\right)^{\perp}$.

Proof. (i) and (iii) follow directly from Lemma 3.1 and (ii) is a consequence of Proposition 2.5.

Our next goal is to describe $\left(\mathbf{e}_{\mu}\right)^{\perp}$ for $\mu \in \mathcal{E}$. Fix $r \geq 1$, and set $C=C_{r}$. By $\mathcal{N}$ we denote the full subcategory of $\operatorname{rep}(C)$ whose objects are the nilpotent representations. Note that $\mathcal{N}$ is an exact abelian subcategory of $\operatorname{rep}(C)$. Let $T$ be a representation in $\mathcal{N}$ having a dense open orbit in $\operatorname{rep}(C, \mathbf{d})$, where $\mathbf{d}=\operatorname{dim} T$. Up to renumbering the vertices of $C$, we may suppose that $d_{r} \leq d_{i}$ for any vertex $i$ of $C$. Then the composition $T\left(\alpha_{r-1}\right) \circ \ldots \circ T\left(\alpha_{1}\right) \circ T\left(\alpha_{r}\right)$ is generically an automorphism. If $d_{r}$ were positive, then $T$ could not be nilpotent. So we see that $d_{r}=0$.

An indecomposable representation $Y$ in $\mathcal{N}$ is uniquely determined by its socle, which is simple and thus corresponds to a vertex $i$ of $C$, and its dimension $l$. Let $\omega$ be the path in $C$ of length $l$ stopping at $i$; it is the shortest path stopping at $i$ with $Y(\omega)=0$. Note that in this way we obtain a bijection from the set of indecomposable representations in $\mathcal{N}$, up to isomorphism, to the set of all paths of positive length in $C$. If $\omega$ is such a path, we let $Y_{\omega}$ be the corresponding indecomposable. 
The following lemma is not difficult; its proof is left to the reader. By $W^{*}$ we denote the dual of the vector space $W$.

Lemma 3.4. Let $\omega$ be a path of positive length in $C$. Then we have for any representation $X$ of $C$ :

$$
\operatorname{Hom}_{C}\left(X, Y_{\omega}\right) \simeq(\operatorname{Coker} X(\omega))^{*} \quad \text { and } \quad \operatorname{Ext}_{C}^{1}\left(X, Y_{\omega}\right) \simeq(\operatorname{Ker} X(\omega))^{*} .
$$

We obtain the following consequence.

Corollary 3.5. Let $\omega$ be a path of positive length in $C$, let $T$ be a representation in $\mathcal{N}$ with ${ }^{1}[T, T]=0$, and set $\mathbf{d}=\operatorname{dim} T$. Then we have:

(i) $Y_{\omega} \in \mathbf{d}^{\perp}$ if and only if $d_{x} \geq d_{h \omega}=d_{t \omega}$ for all vertices of $\omega$,

(ii) $Y_{\omega}$ is a simple object in $\mathbf{d}^{\perp}$ if and only if $d_{x}>d_{h \omega}=d_{t \omega}$ for all inner vertices of $\omega$.

Here we set $t \omega=t \beta_{1}$ and $h \omega=h \beta_{t}$ for $\omega=\beta_{t} \cdots \beta_{1}$, and we call $x$ a vertex of $\omega$ if $x$ is a tail or a head of some $\beta_{i}$. An inner vertex of $\omega$ is a vertex of the form $t \beta_{i}$, $i>1$.

Proof. (i) From Lemma 3.4 we see that $Y_{\omega} \in \mathbf{d}^{\perp}$ if and only if $T(\omega)$ is an isomorphism and therefore $d_{h \omega}=d_{t \omega}$. As $T(\omega)$ factors through $T(x)$ for any vertex $x$ of $\omega$, we find $d_{x} \geq d_{t \omega}=d_{h \omega}$. Conversely, this condition implies that $T(\omega)$, which is a composition of generic maps, one for each arrow $\beta_{i}$, is an isomorphism.

(ii) Assume $Y_{\omega}$ is simple and there is an inner vertex $x$ with $d_{t \omega}=d_{x}=d_{h \omega}$. Denote by $\omega^{\prime}$ the subpath of $\omega$ from $x$ to $h \omega$. By (i), $Y_{\omega^{\prime}}$ belongs to $\mathbf{d}^{\perp}$, and clearly $Y_{\omega^{\prime}}$ is a proper subrepresentation of $Y_{\omega}$. Conversely, a representation $Y_{\omega}$ with $d_{x}>d_{h \omega}=d_{t \omega}$ for all inner vertices cannot have any proper subrepresentation in $\mathbf{d}^{\perp}$, again by (i).

Proposition 3.6. Let $T$ be a representation in $\mathcal{N}$ with ${ }^{1}[T, T]=0$, and set $\mathbf{d}=$ $\operatorname{dim} T$. Then $\mathbf{d}^{\perp_{\mathcal{N}}}$ is an abelian category with $\#(C)_{0}-\sigma(T)=r-\sigma(T)$ simple objects.

Proof. Let $\widehat{C}$ be the quiver obtained from $C=C_{r}$ by deleting the arrow $\alpha_{r}$; it is an $\mathbb{A}_{r}$-quiver. Recall that $T$ in fact lies in $\operatorname{rep}(\widehat{C}, \mathbf{d})$ as $d_{r}=0$. Our strategy is to show that $\mathbf{d}^{\perp_{\mathcal{N}}}$ and $\mathbf{d}^{\perp_{\widehat{C}}}$ have the same simple objects. Then our result follows from Schofield's result [12, Thm. 2.5], as $\widehat{C}$ is a quiver of finite representation type and thus $\mathbf{d}$ is prehomogeneous when viewed as a dimension vector for $\widehat{C}$.

For $Y \in \operatorname{rep}(\widehat{C})$, we have

$$
\operatorname{Hom}_{C}(T, Y)=\operatorname{Hom}_{\widehat{C}}(T, Y) \quad \text { and } \quad\langle\mathbf{d}, \operatorname{dim} Y\rangle_{C}=\langle\mathbf{d}, \operatorname{dim} Y\rangle_{\widehat{C}},
$$

as $d_{r}=0$. We conclude that $\mathbf{d}^{\perp_{\widehat{C}}}=\mathbf{d}^{\perp_{\mathcal{N}}} \cap \operatorname{rep}(\widehat{C})$. Let $Y_{\omega}$ be a simple object of $\mathbf{d}^{\perp \mathcal{N}}$. By Corollary 3.5, the vertex $r$ cannot be an inner vertex of $\omega$, as $d_{r}=0$. Then $Y_{\omega}\left(\alpha_{r}\right)=0$ by the definition of $Y_{\omega}$, and hence $Y_{\omega} \in \operatorname{rep}(\widehat{C})$.

Proposition 3.7. Let $Q$ be an extended Dynkin quiver and $\mathbf{d} \in \mathbb{N}^{Q_{0}}$ a nonprehomogeneous dimension vector with canonical decomposition $\mathbf{d}=p \cdot \mathbf{h}+\mathbf{e}, p \geq 1$. If either $p \geq 2$ or $Q$ is an $\widetilde{\mathbb{A}}_{n-1}$-quiver, the algebra $k[\operatorname{rep}(Q, \mathbf{d})]^{\mathrm{SL}(\mathbf{d})}$ is a polynomial ring in $n+p-1-\sigma(V)$ variables, where $V \in \operatorname{rep}(Q$, e) has an open orbit. 
Proof. The main theorem of Skowroński and Weyman in [17] says that, under our assumptions, $k[\operatorname{rep}(Q, \mathbf{d})]^{\mathrm{SL}(\mathbf{d})}$ is the quotient of a polynomial ring $k\left[c_{0}, \ldots, c_{p}, f_{Y}\right]$ by an ideal generated by $\# \mathcal{E}$ relations, each allowing for the cancellation of one of the $c_{i}$ 's from the list of generators, where $Y$ ranges over the simple non-homogeneous objects in $\mathbf{d}^{\perp}$. Indeed, by Lemma 3.2 and Corollary 3.5, the simple objects in $\mathcal{R}_{\mu}$ correspond bijectively to the "admissible arcs" of 17 . The number of simple objects of $\mathbf{d}^{\perp}$ which belong to $\mathcal{R}_{\mu}$ is $r_{\mu}-\sigma\left(V_{\mu}\right)$, where $V=\bigoplus_{\mu \in \mathcal{E}} V_{\mu}$, by Proposition 3.6. So the number of simple non-homogeneous objects in $\mathbf{d}^{\perp}$ equals

$$
\sum_{\mu \in \mathcal{E}}\left(r_{\mu}-\sigma\left(V_{\mu}\right)\right)=\sum_{\mu \in \mathcal{E}} r_{\mu}-\sigma(V)=n-2+\# \mathcal{E}-\sigma(V)
$$

as $\sum_{\mu \in \mathcal{E}}\left(r_{\mu}-1\right)=n-2$. Taking into account the $\# \mathcal{E}$ relations, we conclude that $k[\operatorname{rep}(Q, \mathbf{d})]^{\operatorname{SL}(\mathbf{d})}$ is a polynomial ring on

$$
(p+1)+(n-2+\# \mathcal{E}-\sigma(V))-\# \mathcal{E}=p+n-1-\sigma(V)
$$

generators.

\section{Proof of the theorem}

4.1. We recall the notation and assumptions for our theorem and keep them fixed throughout this section: $Q$ is an extended Dynkin quiver with $\# Q_{0}=n$, not an oriented cycle, $\mathbf{d} \in \mathbb{N}^{Q_{0}}$ is a non-prehomogeneous dimension vector with canonical decomposition $\mathbf{d}=p \cdot \mathbf{h}+\mathbf{e}, V=\bigoplus_{i=1}^{\sigma(V)} V_{i}^{\lambda_{i}}$ is a representation in the open orbit of $\operatorname{rep}(Q, \mathbf{e})$ with $V_{i}$ indecomposable and pairwise non-isomorphic. We assume that either $Q$ is of type $\widetilde{\mathbb{A}}_{n-1}$ or else $p \geq 3$ and $\lambda_{i} \geq 3$ for $i=1, \ldots, \sigma(V)$. Note that by Proposition 2.1, the variety $\mathcal{Z}_{Q, \mathbf{d}}$ of common zeros of all non-constant semiinvariants has the following description:

$$
\begin{aligned}
\mathcal{Z}_{Q, \mathbf{d}} & =\left\{X \in \operatorname{rep}(Q, \mathbf{d}) ;[X, Y] \neq 0 \text { for all } Y \in \mathbf{d}^{\perp}, Y \neq 0\right\} \\
& =\left\{X \in \operatorname{rep}(Q, \mathbf{d}) ;{ }^{1}[X, Y] \neq 0 \text { for all } Y \in \mathbf{d}^{\perp}, Y \neq 0\right\} .
\end{aligned}
$$

The next result is an immediate consequence of Lemma 3.1 and Proposition 3.3 .

Proposition 4.1. Any representation $X$ in $\mathcal{Z}_{Q, \mathbf{d}}$ has a nonzero preprojective (and a preinjective) direct summand.

The following corollary implies part (ii) of our theorem.

Corollary 4.2. Any irreducible component $\mathcal{C}$ of $\mathcal{Z}_{Q, \mathbf{d}}$ is the closure of some orbit $\mathrm{GL}(\mathbf{d}) \star X$.

We call $X \in \mathcal{Z}_{Q, \mathbf{d}}$ generic if $\overline{\mathrm{GL}(\mathbf{d}) \star X}$ is an irreducible component of $\mathcal{Z}_{Q, \mathbf{d}}$.

Proof. Otherwise, $\mathcal{C}$ contains an infinite number of distinct orbits of maximal dimension, none of which belong to any other irreducible component of $\mathcal{Z}_{Q, \mathbf{d}}$. Some must be given by representations having a direct summand from $\mathcal{R}_{\mu}, \mu \notin \mathcal{E}$. If $X=X_{1} \oplus X_{2} \oplus X_{3}$ is one of them with $X_{1} \neq 0$ preprojective and $X_{2} \in \mathcal{R}_{\mu}, \mu \notin \mathcal{E}$, there exists a non-split extension

$$
0 \rightarrow X_{1} \rightarrow \tilde{X}_{1} \rightarrow X_{2} \rightarrow 0
$$

by Lemma 3.1. Note that $\widetilde{X}_{1}$ still contains a non-zero preprojective summand, as

$$
\partial\left(\operatorname{dim} \widetilde{X}_{1}\right)=\partial\left(\operatorname{dim} X_{1}\right)+\partial\left(\operatorname{dim} X_{2}\right)<0 .
$$


Therefore $\left[\widetilde{X}_{1}, H_{\nu}\right] \neq 0$ for any $\nu \notin \mathcal{E}$. For $Y \in \mathbf{d}^{\perp}$ simple non-homogeneous, we have ${ }^{1}\left[X_{2}, Y\right]=0$ by Lemma 3.1. Mapping the short exact sequence above to $Y$, we conclude that

$$
\left[\widetilde{X}_{1} \oplus X_{3}, Y\right]=\left[X_{1} \oplus X_{2} \oplus X_{3}, Y\right] .
$$

Hence $\widetilde{X}=\widetilde{X}_{1} \oplus X_{3}$ still belongs to $\mathcal{Z}_{Q, \mathbf{d}}$, and even to $\mathcal{C}$, as $X$ lies in the closure of $\mathrm{GL}(\mathbf{d}) \star \widetilde{X}$. This contradicts the maximality of the dimension of $\mathrm{GL}(\mathbf{d}) \star X$.

We fix a sink $z$ in $Q$, and we define $Q^{\prime}$ as in Section 2.4. Note that generically a representation $T$ in $\operatorname{rep}(Q, \mathbf{d})$ is regular and hence does not contain the simple projective $E_{z}$ as a direct summand and thus $\left[T, E_{z}\right]=0$. But $E_{z}$ does not belong to $\mathbf{d}^{\perp}$ either. We conclude that

$$
\left\langle\mathbf{d}, \varepsilon_{z}\right\rangle=d_{z}-\sum_{j=1}^{s} d_{y_{j}}=\left[T, E_{z}\right]-{ }^{1}\left[T, E_{z}\right]<0,
$$

and therefore we may apply Proposition 2.6. The same arguments yield that either $e_{z}=0$ or else $e_{z}<\sum_{j=1}^{s} e_{y_{j}}$. In either case we have $e_{z^{\prime}}^{\prime}=\sum_{j=1}^{s} e_{y_{j}}-e_{z} \geq 0$.

Proposition 4.3. If $X \in \mathcal{Z}_{Q, \mathbf{d}}$ does not contain the simple projective $E_{z}$ as a direct summand, we have that $X$ is generic in $\mathcal{Z}_{Q, \mathbf{d}}$ if and only if $\mathcal{F} X$ is generic in $\mathcal{Z}_{Q^{\prime}, \mathbf{d}^{\prime}}$. Moreover,

$$
\operatorname{codim}_{\mathrm{rep}(Q, \mathbf{d})} \overline{\mathrm{GL}(\mathbf{d}) \star X}=\operatorname{codim}_{\mathrm{rep}\left(Q^{\prime}, \mathbf{d}^{\prime}\right)} \overline{\mathrm{GL}\left(\mathbf{d}^{\prime}\right) \star \mathcal{F} X} .
$$

Proof. We know that $X \in(\operatorname{rep}(Q))^{\prime}$ and $\mathcal{F} X \in\left(\operatorname{rep}\left(Q^{\prime}\right)\right)^{\prime}$. The sets

$$
\operatorname{rep}(Q, \mathbf{d})^{\prime}=\operatorname{rep}(Q, \mathbf{d}) \cap(\operatorname{rep}(Q))^{\prime}, \quad \operatorname{rep}\left(Q^{\prime}, \mathbf{d}^{\prime}\right)^{\prime}=\operatorname{rep}\left(Q^{\prime}, \mathbf{d}^{\prime}\right) \cap\left(\operatorname{rep}\left(Q^{\prime}\right)\right)^{\prime}
$$

are open as $\left[-, E_{z}\right]=0$ and $\left[E_{z^{\prime}}^{\prime},-\right]=0$ are open conditions. Moreover, they are related by a fiber bundle construction [6]. In particular, there is a bijection compatible with $\mathcal{F}$ between the $\operatorname{GL}(\mathbf{d})$-orbits in $\operatorname{rep}(Q, \mathbf{d})^{\prime}$ and the $\operatorname{GL}\left(\mathbf{d}^{\prime}\right)$-orbits in $\operatorname{rep}\left(Q^{\prime}, \mathbf{d}^{\prime}\right)^{\prime}$, preserving their codimensions, closures and inclusions between closures. Hence the claim follows from Proposition 2.6.

4.2. By $\bar{Q}$ we denote the full subquiver of $Q$ with vertex set $Q_{0} \backslash\{z\}$, and we set $\overline{\mathbf{d}}=\left.\mathbf{d}\right|_{\bar{Q}_{0}}$. For $X \in \operatorname{rep}(Q)$, we denote by $\bar{X} \in \operatorname{rep}(\bar{Q})$ the restriction of $X$ to $\bar{Q}$. We set

$$
\mathcal{Z}_{Q, \mathbf{d}}^{\prime \prime}=\left\{X \in \mathcal{Z}_{Q, \mathbf{d}} ; E_{z} \text { is a direct summand of } X\right\} .
$$

As a generic $X \in \mathcal{Z}_{Q, \mathbf{d}}$ contains a non-zero preprojective direct summand and as any indecomposable preprojective representation becomes simple projective under a suitable series of reflection functors, part (i) of our theorem will follow if we show that

$$
\operatorname{codim}_{\mathrm{rep}(Q, \mathbf{d})} \mathcal{Z}_{Q, \mathbf{d}}^{\prime \prime}=n+p-1-\sigma(V) .
$$

Proposition 4.4. The map

$$
\operatorname{rep}(Q, \mathbf{d}) \rightarrow \operatorname{rep}(\bar{Q}, \overline{\mathbf{d}}) \times \operatorname{Mat}\left(d_{z} \times\left(\sum_{j=1}^{s} d_{y_{j}}\right), k\right)
$$

sending $X$ to $\left(\bar{X},\left(X\left(\alpha_{1}\right) \cdots X\left(\alpha_{s}\right)\right)\right)$ restricts to an isomorphism

$$
\mathcal{Z}_{Q, \mathbf{d}}^{\prime \prime} \simeq \mathcal{Z}_{\bar{Q}, \overline{\mathbf{d}}} \times \mathcal{M}
$$

where $\mathcal{M}=\left\{A \in \operatorname{Mat}\left(d_{z} \times\left(\sum_{j=1}^{s} d_{y_{j}}\right), k\right) ; \operatorname{rank} A<d_{z}\right\}$. 
Proof. Indeed, $X \in \operatorname{rep}(Q, \mathbf{d})$ belongs to $\mathcal{Z}_{Q, \mathbf{d}}^{\prime \prime}$ if and only if $X \simeq X^{\prime} \oplus E_{z}$ and $\left[X^{\prime}, Y\right] \neq 0$ for all non-zero $Y \in \mathbf{d}^{\perp}$ with $Y(z)=0$. By Corollary 2.4, these are exactly the objects of $(\overline{\mathbf{d}})^{\perp}$ in $\operatorname{rep}(\bar{Q}, \overline{\mathbf{d}})$, extended by 0 to $Q$. But if $Y(z)=0$, we have $\left[X^{\prime}, Y\right]=[\bar{X}, Y]$. The result follows.

Let $\bar{H}$ be a representation in the open orbit of $\operatorname{rep}(\bar{Q}, \overline{\mathbf{h}})$, and note that the image $\bar{H}_{\mu}$ of the simple homogeneous representation $H_{\mu}$ is isomorphic to $\bar{H}$ for any $\mu \notin \mathcal{E}$. We will need the following lemma.

Lemma 4.5. $\quad$ (i) $\sigma(\bar{H}) \leq 3 h_{z}-2=3\left(h_{z}-1\right)+1$.

(ii) If either $Q$ is an $\widetilde{\mathbb{A}}_{n-1}$-quiver or else $V=\bigoplus V_{i}^{\lambda_{i}}$ with $\lambda_{i} \geq 3$ for all $i$, then $\sigma(\bar{V}) \leq \sigma(V)+e_{z^{\prime}}^{\prime}$.

Proof. (i) Clearly $\bar{H}$ has at most as many pairwise non-isomorphic direct summands as $\sum_{j=1}^{s} \operatorname{dim}_{k} H_{\mu}\left(y_{j}\right), \mu \notin \mathcal{E}$, which implies that

$$
\sigma(\bar{H}) \leq \sum_{j=1}^{s} h_{y_{j}}=2 h_{z} .
$$

The last equality follows from

$$
0=\left\langle\varepsilon_{z}, \mathbf{h}\right\rangle+\left\langle\mathbf{h}, \boldsymbol{\varepsilon}_{z}\right\rangle=h_{z}+h_{z}-\sum_{j=1}^{s} h_{y_{j}} .
$$

This yields our claim except in the case $h_{z}=1$. But in that case, $\bar{Q}$ is a Dynkin quiver, and we have

$$
[\bar{H}, \bar{H}]=\langle\overline{\mathbf{h}}, \overline{\mathbf{h}}\rangle_{\bar{Q}}=\langle\overline{\mathbf{h}}, \overline{\mathbf{h}}\rangle_{Q}=\left\langle\mathbf{h}-\varepsilon_{z}, \mathbf{h}-\varepsilon_{z}\right\rangle=\left\langle\varepsilon_{z}, \boldsymbol{\varepsilon}_{z}\right\rangle=1 .
$$

Recall that ${ }^{1}[\bar{H}, \bar{H}]=0$ as the orbit of $\bar{H}$ is open. In particular, $\bar{H}$ is indecomposable and $\sigma(\bar{H})=1$.

(ii) We restrict $V$ to the support of $\mathbf{e}$, which is a tame quiver $K$ for which $\mathbf{e}$ is a sincere dimension vector. If $e_{z}=0$, i.e. if $z$ is not a vertex of $K$, we have $\bar{K}=K$, $\bar{V}=V$ and $e_{z^{\prime}}^{\prime} \geq 0$. Otherwise, $z$ is a sink of $K$, and we can apply our results from [9]: $\mathcal{Z}_{K, \mathbf{e}}$ is a complete intersection of codimension $\# K_{0}-\sigma(V)$, and $\mathcal{Z}_{\bar{K}, \overline{\mathbf{e}}}$ is a complete intersection of codimension $\# K_{0}-1-\sigma(\bar{V})$. Note that either every connected component of $K$ is a quiver of $\mathbb{A}$-type or else the multiplicity $\lambda_{i}$ of every indecomposable $\left.V_{i}\right|_{K}$ arising in $\left.V\right|_{K}$ is at least 3. Set

$$
\mathcal{Z}_{K, \mathbf{e}}^{\prime \prime}=\left\{X \in \mathcal{Z}_{K, \mathbf{e}} ;\left[X, E_{z}\right] \neq 0\right\}
$$

and remember that, as in Proposition 4.4, $\mathcal{Z}_{K, \mathbf{e}}^{\prime \prime} \stackrel{\sim}{\longrightarrow} \mathcal{Z}_{\bar{K}, \overline{\mathbf{e}}} \times \mathcal{M}^{\prime}$ with

$$
\mathcal{M}^{\prime}=\left\{B \in \operatorname{Mat}\left(e_{z} \times \sum_{j=1}^{s} e_{y_{j}}, k\right) ; \operatorname{rank}(B)<e_{z}\right\} .
$$

We conclude that

$$
\begin{aligned}
\# K_{0}-\sigma(V) & =\operatorname{codim}_{\mathrm{rep}(K, \mathbf{e})} \mathcal{Z}_{K, \mathbf{e}} \leq \operatorname{codim}_{\mathrm{rep}(K, \mathbf{e})} \mathcal{Z}_{K, \mathbf{e}}^{\prime \prime} \\
& =\# K_{0}-1-\sigma(\bar{V})+e_{z^{\prime}}^{\prime}+1,
\end{aligned}
$$

as $\operatorname{codim} \mathcal{M}^{\prime}=\sum_{j=1}^{s} e_{y_{j}}-e_{z}+1=e_{z^{\prime}}^{\prime}+1$. The result follows. 
We are now ready to finish the proof of part (i) of our theorem. We need to show that

$$
\operatorname{codim}_{\mathrm{rep}(Q, \mathbf{d})} \mathcal{Z}_{Q, \mathbf{d}}^{\prime \prime} \geq n+p-1-\sigma(V) .
$$

Note that $\bar{Q}$ is a disjoint union of Dynkin quivers; in fact it is an $\mathbb{A}_{n-1}$-quiver if $Q$ is an $\widetilde{\mathbb{A}}_{n-1}$-quiver. In the remaining cases, the multiplicity of any indecomposable direct summand in the representation $\bar{H}^{p} \oplus \bar{V} \in \operatorname{rep}(\bar{Q}, \overline{\mathbf{d}})$, whose $\operatorname{GL}(\overline{\mathbf{d}})$-orbit is open, is at least 3 . Therefore we know from $[9$ that

$$
\operatorname{codim} \mathcal{Z}_{\bar{Q}, \overline{\mathbf{d}}}=n-1-\sigma\left(\bar{V} \oplus \bar{H}^{p}\right) .
$$

We compute, using Proposition 4.4, the preceding lemma, and [9] for $(\bar{Q}, \overline{\mathbf{d}})$ :

$$
\begin{aligned}
\operatorname{codim}_{\mathrm{rep}(Q, \mathbf{d})} \mathcal{Z}_{Q, \mathbf{d}}^{\prime \prime}-(n+p-1-\sigma(V)) \\
=\operatorname{codim}_{\operatorname{rep}(\bar{Q}, \overline{\mathbf{d}})} \mathcal{Z}_{\bar{Q}, \overline{\mathbf{d}}}+\left(d_{z^{\prime}}^{\prime}+1\right)-(n+p-1-\sigma(V)) \\
=\left(n-1-\sigma\left(\bar{V} \oplus \bar{H}^{p}\right)\right)+\left(p h_{z}+e_{z^{\prime}}^{\prime}+1\right)-(n+p-1-\sigma(V)) \\
=(\sigma(\bar{V})+\sigma(\bar{H})-\sigma(\bar{V} \oplus \bar{H}))+\left(p\left(h_{z}-1\right)+1-\sigma(\bar{H})\right) \\
\quad+\left(\sigma(V)+e_{z^{\prime}}^{\prime}-\sigma(\bar{V})\right) \geq 0 .
\end{aligned}
$$

In the last sum, each summand is non-negative. This is obvious for the first one, and it follows from the preceding lemma for the second and the third. Note that either $p \geq 3$ or else $Q$ is an $\widetilde{\mathbb{A}}_{n-1}$-quiver and $h_{z}=1$.

4.3. Finally, we prove part (iii) of the theorem.

Lemma 4.6. For $\mathbf{d}=p \mathbf{h}+\mathbf{e}, p \geq 3, \mathcal{Z}_{Q, \mathbf{d}}$ contains a generic representation $X=X_{\mathcal{P}} \oplus X_{\mathcal{R}} \oplus X_{\mathcal{I}}$ with $X_{\mathcal{P}}$ preprojective, $X_{\mathcal{R}}$ regular, and $X_{\mathcal{I}}$ preinjective such that the preprojective part $X_{\mathcal{P}}$ has defect $\partial\left(X_{\mathcal{P}}\right)=-1$.

Note that in particular $X_{\mathcal{P}}$ is indecomposable.

Proof. We assume there is a sink $z$ with $h_{z}=1$. If no such sink exists, there is a source $y$ with $h_{y}=1$, and applying the dual arguments we find that $\partial\left(X_{\mathcal{I}}\right)=1$, which implies $\partial\left(X_{\mathcal{P}}\right)=-1$.

We start from a representation $W$ with dimension vector $p \mathbf{h}$ which is a direct sum of all simple regular non-homogeneous representations and some simple homogeneous representations (if necessary). Obviously there is an exact sequence in $\operatorname{rep}(Q)$ of the form

$$
0 \rightarrow E_{z} \rightarrow W \rightarrow W^{\prime} \rightarrow 0
$$

for some $W^{\prime}$. Then no indecomposable direct summand of $W^{\prime}$ is preprojective, by Lemma 3.1 Let $X^{\prime}=E_{z} \oplus W^{\prime}$. Thus the defect of the preprojective part $E_{z}$ of $X^{\prime}$ equals $\left\langle\mathbf{h}, \varepsilon_{z}\right\rangle=-h_{z}=-1$. Observe that $\left[X^{\prime}, Y\right] \neq 0$ for any regular repesentation $Y \neq 0$. Indeed, if $Y$ is simple homogeneous, then $\left[X^{\prime}, Y\right] \geq\left[E_{z}, Y\right]>0$, and if $Y$ is simple non-homogeneous, then $\left[X^{\prime}, Y\right] \geq[W, Y]>0$. Thus $X^{\prime}$ lies in $\mathcal{Z}_{Q, \mathbf{d}}$.

$X^{\prime}$ belongs to $\overline{\mathrm{GL}(\mathbf{d}) \star X}$ for some generic $X$, which we decompose as $X=$ $X_{\mathcal{P}} \oplus X_{\mathcal{R}} \oplus X_{\mathcal{I}}$ with $X_{\mathcal{P}}$ preprojective, $X_{\mathcal{R}}$ regular, and $X_{\mathcal{I}}$ preinjective. By Proposition 4.1 we know that $X_{\mathcal{P}} \neq 0$ and thus $\partial\left(X_{\mathcal{P}}\right) \leq-1$. Choose $\nu \in \mathbb{P}^{1}(k) \backslash \mathcal{E}$ in such a way that $H_{\nu}$ is not a direct summand of $X$ nor of $X^{\prime}$, and remember that $\left[X, H_{\nu}\right] \leq\left[X^{\prime}, H_{\nu}\right]$. Using Lemma 3.1 we compute:

$$
\begin{aligned}
1 & \leq-\partial\left(X_{\mathcal{P}}\right)=\left\langle\operatorname{dim} X_{\mathcal{P}}, \mathbf{h}\right\rangle=\left[X_{\mathcal{P}}, H_{\nu}\right]=\left[X, H_{\nu}\right] \\
\leq\left[X^{\prime}, H_{\nu}\right] & =-\partial\left(X_{\mathcal{P}}^{\prime}\right)=1 .
\end{aligned}
$$


For each natural number $r$, the vector $\operatorname{dim} X_{\mathcal{P}}+r \mathbf{h}$ is the dimension vector of some indecomposable representation $X_{\mathcal{P}}[r]$, which is still preprojective with defect -1 and thus has an open orbit in $\operatorname{rep}\left(Q, \operatorname{dim} X_{\mathcal{P}}+r \mathbf{h}\right)$. For $s \in \mathbb{N}$ we let $X_{\mathcal{I}}[s]$ be an indecomposable representation with $\operatorname{dim} X_{\mathcal{I}}[s]=\operatorname{dim} X_{\mathcal{I}}+s \mathbf{h}$. Set $X[r, s]=$ $X_{\mathcal{P}}[r] \oplus X_{\mathcal{R}} \oplus X_{\mathcal{I}}[s]$. The following result implies part (iii) of our theorem:

Proposition 4.7. The representations $X[r, s]$ are pairwise non-isomorphic and

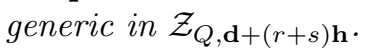

Proof. Choose $Y \in(\mathbf{d}+(r+s) \mathbf{h})^{\perp}=\mathbf{d}^{\perp}$, and remember that $Y$ is regular and has defect 0 . Therefore we have

$$
\left[X_{\mathcal{P}}[r], Y\right]=\left\langle\operatorname{dim} X_{\mathcal{P}}[r], \operatorname{dim} Y\right\rangle=\left\langle\operatorname{dim} X_{\mathcal{P}}, \operatorname{dim} Y\right\rangle=\left[X_{\mathcal{P}}, Y\right],
$$

and thus

$$
[X[r, s], Y]=\left[X_{\mathcal{P}}, Y\right]+\left[X_{\mathcal{R}}, Y\right]=[X, Y]>0
$$

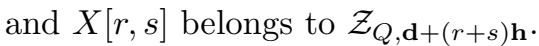

In order to show that $X[r, s]$ is generic, it is enough to prove

$$
{ }^{1}[X[r, s], X[r, s]]=n+p+r+s-1-\sigma(V),
$$

which follows if

$$
{ }^{1}[X[r, s], X[r, s]]={ }^{1}[X, X]+r+s .
$$

As above we have

$$
{ }^{1}\left[X_{\mathcal{R}}, X_{\mathcal{P}}[r]\right]={ }^{1}\left[X_{\mathcal{R}}, X_{\mathcal{P}}\right] \quad \text { and } \quad{ }^{1}\left[X_{\mathcal{I}}[s], X_{\mathcal{R}}\right]={ }^{1}\left[X_{\mathcal{I}}, X_{\mathcal{R}}\right] .
$$

We compute

$$
\begin{aligned}
{ }^{1}\left[X_{\mathcal{I}}[s], X_{\mathcal{P}}[r]\right] & =-\left\langle\operatorname{dim} X_{\mathcal{I}}[s], \operatorname{dim} X_{\mathcal{P}}[r]\right\rangle \\
& =-\left\langle\operatorname{dim} X_{\mathcal{I}}+s \mathbf{h}, \operatorname{dim} X_{\mathcal{P}}+r \mathbf{h}\right\rangle \\
& ={ }^{1}\left[X_{\mathcal{I}}, X_{\mathcal{P}}\right]-s \partial\left(\operatorname{dim} X_{\mathcal{P}}\right)+r \partial\left(\operatorname{dim} X_{\mathcal{I}}\right) \\
& ={ }^{1}\left[X_{\mathcal{I}}, X_{\mathcal{P}}\right]+s+r .
\end{aligned}
$$

By Lemma 3.1 these are the only terms that do not vanish.

\section{THE ORIENTED CYCLE}

5.1. In this section we wish to generalize our results to the only extended Dynkin quiver not considered so far, the oriented cycle, i.e. the quiver $Q$ with $Q_{0}=$ $\{1,2, \ldots, n\}$ and $Q_{1}=\left\{\alpha_{i}: i \rightarrow(i+1) ; i \in Q_{0}\right\}$; we view the elements of $Q_{0}$ as representatives of $\mathbb{Z} / n \mathbb{Z}$. The category $\operatorname{rep}(Q)$ of finite dimensional representations of $Q$ decomposes into a family $\coprod_{\mu \in k} \mathcal{R}_{\mu}$ of uniserial categories $\mathcal{R}_{\mu}$ parametrized by $\mu \in k$. For $\mu \neq 0, \mathcal{R}_{\mu}$ contains a unique simple representation $H_{\mu}$ with $\operatorname{dim} H_{\mu}=\mathbf{h}$, where $h_{i}=1$ for $i \in Q_{0}$. For $\mu=0, \mathcal{R}_{0}$ consists of all nilpotent representations (compare Section 3.2). If $n \geq 2$, its simple objects are just the one-dimensional representations of $Q$.

We recall the description of the semi-invariants for $\operatorname{rep}(Q, \mathbf{d})$ from [10] and [17. For $\mathbf{d} \in \mathbb{N}^{Q_{0}}$, we may assume that $d_{1}=p \leq d_{i}, i \in Q_{0}$, up to renumbering the vertices of $Q$. For $X \in \operatorname{rep}(Q, \mathbf{d})$, the coefficients $c_{1}(X), \ldots, c_{p}(X)$ of the characteristic polynomial

$$
\operatorname{det}\left(T-X\left(\alpha_{n}\right) \cdot \ldots \cdot X\left(\alpha_{1}\right)\right)=T^{p}+c_{1}(X) T^{p-1}+\cdots+c_{p}(X)
$$


are clearly invariant under $\mathrm{GL}(\mathbf{d})$. For two integers $i<j \leq i+n$, the path $\alpha_{j-1} \cdots \alpha_{i}$ is called an admissible arc $A=[i, j]$ if $d_{i}=d_{j}<d_{m}$ for all $m$ with $i<m<j$. For any admissible arc $A=[i, j]$ the determinant

$$
f_{A}(X)=\operatorname{det}\left(X\left(\alpha_{j-1}\right) \cdot \ldots \cdot X\left(\alpha_{i}\right)\right)
$$

is a semi-invariant. We call an admissible arc $B=[i, j]$ minimal if $d_{i}=d_{j}=p$.

Proposition 5.1. Let $Q$ be the oriented cycle, and let $\mathbf{d} \in \mathbb{N}^{Q_{0}}$ with $d_{1}=p \leq d_{i}$ for all $i \in Q_{0}$.

(i) The algebra of semi-invariants is the polynomial algebra

$$
k[\operatorname{rep}(Q, \mathbf{d})]^{\mathrm{SL}(\mathbf{d})}=k\left[c_{1}, \ldots, c_{p} ;\left\{f_{A}\right\}\right] /\left(\prod f_{B}-c_{p}\right),
$$

where $A$ ranges over all admissible and $B$ over all minimal admissible arcs of $Q$.

(ii) The algebra $k[\operatorname{rep}(Q, \mathbf{d})]^{\mathrm{GL}(\mathbf{d})}$ of $\mathrm{GL}(\mathbf{d})$-invariants is a polynomial ring in $c_{1}, \ldots, c_{p}$.

Proof. Both statements are essentially contained in [17]: (i) is stated explicitly, and (ii) is the fact that an invariant is a semi-invariant with trivial weight.

Theorem 2. Let $Q$ be the oriented cycle, $\mathbf{d} \in \mathbb{N}^{Q_{0}}$ with $d_{1}=p \leq d_{i}$ for all $i \in Q_{0}$.

(i) The set $\mathcal{Z}_{Q, \mathbf{d}}$ of common zeros of $c_{1}, \ldots, c_{p} ;\left\{f_{A}\right\}$ is a complete intersection, where $A$ ranges over all admissible arcs of $Q$.

(ii) The set $\mathcal{N}_{\mathbf{d}}$ of nilpotent representations in $\operatorname{rep}(Q, \mathbf{d})$ is the set of common zeros of $c_{1}, \ldots, c_{p}$; it is a complete intersection.

As nilpotent representations do not depend on parameters, any irreducible component of $\mathcal{Z}_{Q, \mathbf{d}}$ or $\mathcal{N}_{\mathbf{d}}$ is the closure of an orbit. The number of irreducible components of $\mathcal{Z}_{Q, \mathbf{d}}$ can be shown to be bounded for the oriented cycle. Note that as a consequence of our two theorems, we obtain that $\mathcal{Z}_{Q, \mathbf{d}}$ is a complete intersection for any $\widetilde{\mathbb{A}}_{n-1}$-quiver.

5.2. Our strategy is to compare $\operatorname{rep}(Q, \mathbf{d})$ with $\operatorname{rep}(\widetilde{Q}, \widetilde{\mathbf{d}})$, where

$$
\widetilde{Q}=\underbrace{1 \stackrel{\alpha_{1}}{\longrightarrow} 2-\cdots \rightarrow n \stackrel{\alpha_{n}}{\longrightarrow} 0}_{\beta} \gamma
$$

and

$$
\widetilde{d}_{i}= \begin{cases}d_{i} & 1 \leq i \leq n, \\ p=d_{1} & i=0 .\end{cases}
$$

For $Y \in \operatorname{rep}(\widetilde{Q}, \widetilde{\mathbf{d}})$ the coefficients $\widetilde{c}_{0}(Y), \ldots, \widetilde{c}_{p}(Y)$ of the polynomial

$$
\operatorname{det}\left(Y(\beta) T-Y\left(\alpha_{n}\right) \cdot \ldots \cdot Y\left(\alpha_{1}\right)\right)=\widetilde{c}_{0}(Y) T^{p}+\widetilde{c}_{1}(Y) T^{p-1}+\cdots+\widetilde{c}_{p}(Y)
$$

are semi-invariants; note that $\widetilde{c}_{0}(Y)=\operatorname{det}(Y(\beta))$. For every admissible arc $A=$ $[i, j]$ of $Q$ as defined in Section 5.1 , there is a semi-invariant $\widetilde{f}_{A}$ given by

$$
\tilde{f}_{A}(Y)=\operatorname{det}\left(Y\left(\alpha_{j-1}\right) \cdot \ldots \cdot Y\left(\alpha_{i}\right)\right) .
$$

From [17] we know that

$$
k[\operatorname{rep}(\widetilde{Q}, \widetilde{\mathbf{d}})]^{\operatorname{SL}(\widetilde{\mathbf{d}})}=k\left[\widetilde{c}_{1}, \ldots, \widetilde{c}_{p} ;\left\{\widetilde{f}_{A}\right\}\right] /\left(\prod \widetilde{f}_{B}-\widetilde{c}_{p}\right),
$$


where $A$ ranges over all admissible and $B$ over all minimal admissible arcs for $Q$.

We leave the proof of the following lemma to the reader.

Lemma 5.2. The map

$$
\Phi: \operatorname{GL}(p) \times \operatorname{rep}(Q, \mathbf{d}) \rightarrow \operatorname{rep}(\widetilde{Q}, \widetilde{\mathbf{d}})
$$

given by

$$
\Phi(s, X)(\gamma)= \begin{cases}X\left(\alpha_{i}\right) & \gamma=\alpha_{i}, i<n, \\ s \cdot X\left(\alpha_{n}\right) & \gamma=\alpha_{n}, \\ s & \gamma=\beta\end{cases}
$$

is a $\mathrm{GL}(\widetilde{\mathbf{d}})$-equivariant open immersion onto the set

$$
\operatorname{rep}(\widetilde{Q}, \widetilde{\mathbf{d}})^{\prime}=\{Y \in \operatorname{rep}(\widetilde{Q}, \widetilde{\mathbf{d}}) ; \operatorname{det}(Y(\beta)) \neq 0\},
$$

where $\operatorname{GL}(\widetilde{\mathbf{d}})$ acts on $\operatorname{GL}(p) \times \operatorname{rep}(Q, \mathbf{d})$ by

$$
h \star(s, X)=\left(h_{0} s h_{1}^{-1}, \bar{h} \star X\right)
$$

and $\bar{h}_{i}=h_{i}$ for $i \in Q_{0}$.

Note that by definition,

$$
\begin{aligned}
\Phi^{*}\left(\widetilde{c}_{i}\right)(s, X) & = \begin{cases}\operatorname{det}(s) \cdot c_{i}(X) & i=1, \ldots, p, \\
\operatorname{det}(s) & i=0 ;\end{cases} \\
\Phi^{*}\left(\widetilde{f}_{A}\right)(s, X) & = \begin{cases}\operatorname{det}(s) \cdot f_{A}(X) & \text { if } \alpha_{n} \text { belongs to } A, \\
f_{A}(X) & \text { otherwise. }\end{cases}
\end{aligned}
$$

We conclude that the zero set $\mathcal{V}\left(c_{1}, \ldots, c_{p},\left\{f_{A}\right\}\right)=\mathcal{Z}_{Q, \mathbf{d}} \subseteq \operatorname{rep}(Q, \mathbf{d})$ has the same codimension as the zero set $\mathcal{V}\left(\widetilde{c}_{1}, \ldots, \widetilde{c}_{p},\left\{\widetilde{f}_{A}\right\}\right)=\mathcal{Y}_{\widetilde{Q}, \widetilde{\mathbf{d}}} \subseteq \operatorname{rep}(\widetilde{Q}, \widetilde{\mathbf{d}})$. As $\mathcal{Z}_{\widetilde{Q}, \tilde{\mathbf{d}}}=\mathcal{V}\left(\widetilde{c}_{0}\right) \cap \mathcal{Y}_{\widetilde{Q}, \tilde{\mathbf{d}}}$ is a complete intersection by Theorem 1, $\mathcal{Y}_{\widetilde{Q}, \widetilde{\mathbf{d}}}$ is as well. This proves part $(i)$.

As for part (ii), we need to study the set $\mathcal{V}\left(c_{1}, \ldots, c_{p}\right)$ of common zeros of $c_{1}, \ldots, c_{p-1}, c_{p}=\prod f_{B}$, where $B$ ranges over all minimal admissible arcs. As

$$
\mathcal{V}\left(c_{1}, \ldots, c_{p}\right)=\bigcup_{B} \mathcal{V}\left(c_{1}, \ldots, c_{p-1}, f_{B}\right),
$$

this is a complete intersection as well. Clearly, a representation $X$ is nilpotent if and only if the characteristic polynomial of $X\left(\alpha_{n}\right) \cdot \ldots \cdot X\left(\alpha_{1}\right)$ is $T^{p}$.

\section{REFERENCES}

1. I. N. Bernstein, I. M. Gelfand and V. A. Ponomarev, Coxeter functors, and Gabriel's theorem, Russian Math. Surveys 28 (1973), 17-32. MR0393065 (52:13876)

2. C. Chang and J. Weyman, Representations of quivers with free modules of covariants, J. Pure Appl. Algebra 192 (2004), 69-94. MR2067189 (2005g:16022)

3. H. Derksen and J. Weyman, Semi-invariants of quivers and saturation for LittlewoodRichardson coefficients, J. Amer. Math. Soc. 13 (2000), 467-479. MR1758750 (2001g:16031)

4. P. Gabriel, Représentations indécomposables, Séminaire Bourbaki 1973/74, Lecture Notes in Math. 431 (1975), 143-169. MR0485996 (58:5788)

5. H. Kraft, Geometrische Methoden in der Invariantentheorie, Vieweg Verlag, Braunschweig, 1984. MR768181 (86j:14006)

6. H. Kraft and Ch. Riedtmann, Geometry of representations of quivers, London Math. Soc. Lecture Notes 116 (1985), 109-147. MR897322 (88k:16028) 
7. P. Littelmann, Koreguläre und äquidimensionale Darstellungen, J. Algebra 123 (1989), 193222. MR1000484 (90e:20039)

8. Ch. Riedtmann and G. Zwara, On the zero set of semi-invariants for quivers, Ann. Sci. École Norm. Sup. 36 (2003), 969-976. MR2032531 (2005b:16032)

9. Ch. Riedtmann and G. Zwara, On the zero set of semi-invariants for tame quivers, Comment. Math. Helv. 79 (2004), 350-361. MR.2059437 (2005g:16024)

10. C. M. Ringel, The rational invariants of the tame quivers, Invent. Math. 58 (1980), 217-239. MR:571574 (81f:16048)

11. C. M. Ringel, Tame algebras and integral quadratic forms, Springer Lecture Notes in Math. 1099 (1984). MR 774589 (87f:16027)

12. A. Schofield, Semi-invariants of quivers, J. London Math. Soc. 43 (1991), 385-395. MR:1113382 (92g:16019)

13. A. Schofield and M. Van den Bergh, Semi-invariants of quivers for arbitrary dimension vectors, preprint, math.RA/9907174. MR.1908144 (2003e:16016)

14. G. W. Schwarz, Representations of simple Lie groups with regular rings of invariants, Invent. Math. 49 (1978), 167-191. MR511189 (80m:14032)

15. G. W. Schwarz, Representations of simple Lie groups with a free module of covariants, Invent. Math. 50 (1978), 1-12. MR516601 (80c:14008)

16. G. W. Schwarz, Lifting smooth homotopies of orbit spaces, Inst. Hautes Études Sci. Publ. Math. 51 (1980), 37-135. MR573821 (81h:57024)

17. A. Skowroński and J. Weyman, The algebras of semi-invariants of quivers, Transform. Groups 5 (2000), 361-402. MR1800533 (2001m:16017)

Mathematisches Institut, Universität Bern, Sidlerstrasse 5, CH-3012 Bern, SwitzerLAND

E-mail address: christine.riedtmann@math-stat.unibe.ch

Faculty of Mathematics and Computer Science, Nicolaus Copernicus University, Chopina 12/18, 87-100 Toruń, Poland

E-mail address: gzwara@mat.uni.torun.pl 